\title{
OPTICAL PARAMETRIC AMPLIFICATION BY INCOHERENT CONICAL PUMP BEAM
}

\author{
V. Tamulienė, V. Smilgevičius, D. Kudarauskas, R. Butkus, A. Stabinis, and A. Piskarskas \\ Department of Quantum Electronics, Vilnius University, Sauletekio 10, LT-10223 Vilnius, Lithuania \\ E-mail: viktorija.tamuliene@ff.vu.lt
}

Received 9 September 2016; revised 19 October 2016; accepted 21 December 2016

\begin{abstract}
Optical parametric amplification of an input narrowband signal beam in the field of a spatially incoherent conical pump is investigated. Two amplification regimes - onset and exponential - are distinguished. The possibility to amplify the signal beam significantly and obtain a smooth intensity profile at the output is revealed. It is shown that the incoherent conical beam may act on the signal beam as a plane wave. Good agreement of theoretical and experimental data is obtained.
\end{abstract}

Keywords: optical parametric amplification, conical beams, incoherence

PACS: $42.65 .-\mathrm{k}, 42.65 . \mathrm{Yj}, 42.25 . \mathrm{Kb}$

\section{Introduction}

The generation and amplification of coherent radiation by an uncorrelated pump source is an interesting field of research. In the active laser medium pumped by incoherent light, only a narrow part of the spectrum is utilised. By parametric frequency down- and up-conversions, all spectral components of the broadband incoherent pump can be depleted [1].

Successful generation of coherent light in threewave interaction needs fulfilment of a specific condition. In the time domain, it is the group velocity matching between two incoherent waves while the group velocity of the coherent wave must differ [2, 3. In the space domain, the group velocity is substituted by spatial walk-off. Instead of choosing particular crystals at particular wavelengths, the appropriate angular dispersion of signal and pump waves can be used [1]. An effective parametric amplification of a narrowband signal in the field of an incoherent pump can be achieved even in the case of no group velocity matching when the background of the amplified spectrum is filtered out [ [

Instead of incoherent waves, spatially incoherent beams that obey a broadband angular spectrum are often discussed [5]. In contrast to the plane wave, transverse spatial structures are two-dimensional. This also allows composing the conical structures. The Bessel beam [6, d] refers to the coherent conical beam that is composed of a set of beams with correlated phases. The incoherent conical beam may be regarded as a set of mutually uncorrelated beams. Each of them may also be incoherent. The up- [8] and down- [9] conversions of incoherent conical beams allow the generation of a beam with a narrowband angular spectrum. The motivation of studying such interactions is also provided by parametric combining. Indeed, beams that compose a conical beam may be seen as pump beams in the parametric combining process. Their energy may be transferred to a single beam that should be coherent.

Here we focus on an optical parametric amplifier (OPA) of an input narrowband seed which is amplified by a spatially incoherent conical pump. Note that the research of pumping by the Bessel beam revealed new possibilities to control the angular profile of an amplified signal [10]. Here, we reveal new interesting features, namely, that the incoherent conical beam may act on the amplified signal beam as a plane wave giving rise to exponential amplification. Moreover, the amplification is dictated by the pump intensity that 'forgets' the random phases of the pump 
field. A detailed study shows the possibility to reduce the generated noise in the angular and intensity profiles of the amplified signal.

The rest of the paper is organized as follows. In Section 2, we first investigate the properties of the incoherent conical beams that are used in the following sections. Here, we distinguish two amplification regimes: onset of amplification and exponential amplification. Both regimes are studied theoretically as well as numerically. In Section 3, the experimental results are presented that qualitatively agree with the theoretical ones. In Section 4, conclusions are drawn.

\section{Theoretical results}

\subsection{Properties of incoherent conical beam}

Let us find the correlation function of the incoherent conical beam. We assume the homogeneous, isotropic pump field $A_{30}$ that obeys the Gaussian statistics and has an angular spectrum of the following form:

$$
S_{A_{30}}(\beta)=S_{A_{30}}\left(\beta_{0}\right) \exp \left(-\frac{\left(\beta-\beta_{0}\right)^{2}}{\Delta \beta_{3}^{2}}\right) .
$$

Here $\beta_{0}=k_{3} \theta_{0}, k_{3}$ is the pump wave number, $2 \theta_{0}$ is the cone angle. $2 \Delta \beta_{3}$ is the cone width and it was assumed that $\Delta \beta_{3} \ll \beta_{0}$. The correlation function is given by

$$
B_{A_{30}}(r)=\frac{1}{2 \pi} S_{A_{30}}\left(\beta_{0}\right) \int_{0}^{\infty} \beta \exp \left(-\frac{\left(\beta-\beta_{0}\right)^{2}}{\Delta \beta_{3}^{2}}\right) J_{0}(\beta r) \mathrm{d} \beta .
$$

Here $J_{0}$ is the Bessel function. We obtain

$$
B_{A_{30}}(r)=\left\langle I_{3}\right\rangle \exp \left(-\frac{\Delta \beta_{3}^{2} r^{2}}{4}\right) J_{0}\left(\beta_{0} r\right)
$$

where $\left\langle I_{3}\right\rangle=\frac{\beta_{0} \Delta \beta_{3}}{2 \sqrt{\pi}} S_{A_{30}}\left(\beta_{0}\right)$ is the mean intensity of the pump field. As we can see, the correlation radius of the incoherent conical beam at $\beta_{0} \gg \Delta \beta_{3}$ is mainly determined by $\beta_{0}$.

Further, we will need the intensity correlation function $B_{I_{3}}(r)=\left\langle I_{3}\left(r_{1}\right) I_{3}\left(r_{2}\right)\right\rangle$, where $r=\left|r_{1}-r_{2}\right|$. One can obtain

$$
\begin{aligned}
& B_{I_{3}}(r)=\left\langle A_{30}\left(r_{1}\right) A_{30}^{*}\left(r_{1}\right) A_{30}\left(r_{2}\right) A_{30}^{*}\left(r_{2}\right)\right\rangle \\
& =\left\langle I_{3}\right\rangle^{2}+\left|B_{A_{30}}(r)\right|^{2} \\
& =\left\langle I_{3}\right\rangle^{2}\left[1+\exp \left(-\frac{\Delta \beta_{3}^{2} r^{2}}{2}\right) J_{0}^{2}\left(\beta_{0} r\right)\right] .
\end{aligned}
$$

Now, let us find the correlation function $B_{I_{f}}$ as well as the spectrum of the product $I_{e}=A_{10}(r) I_{3}(r)$ that appears in the governing equations in the case of the onset of parametric amplification. Here $A_{10}$ denotes the axial input signal beam,

$$
A_{10}(\rho)=a_{0} \exp \left(-\frac{\rho^{2}}{d_{1}^{2}}\right),
$$

where $a_{0}$ is the amplitude, $d_{1}$ is the input beam radius, and $\rho=\sqrt{x^{2}+y^{2}},(x, y, z)$ are the Cartesian coordinates. The correlation function of the input signal beam is given by

$$
\begin{aligned}
& B_{A_{10}}(r)=\int_{0}^{\infty} \rho A_{10}(\rho+r / 2) A_{10}^{*}(\rho-r / 2) \mathrm{d} \rho \\
& =B_{A_{10}}(0) \exp \left(-\frac{r^{2}}{2 d_{1}^{2}}\right),
\end{aligned}
$$

where $B_{A_{10}}(0)=\frac{a_{0}^{2} d_{1}^{2}}{4}$. We assume that at the input the signal and pump beams are uncorrelated. Then

$$
B_{I_{e}}=B_{A_{10}} B_{I_{3}} .
$$

By the use of Eqs. (4) and (6) we obtain

$$
\begin{aligned}
& B_{I_{e}}(r) \\
& =\left\langle I_{e}\right\rangle^{2}\left[\exp \left(-\frac{r^{2}}{2 d_{1}^{2}}\right)+\exp \left(-\frac{r^{2} \Delta \beta_{n}^{2}}{2}\right) J_{0}^{2}\left(\beta_{0} r\right)\right],
\end{aligned}
$$

where $\left\langle I_{e}\right\rangle^{2}=\frac{a_{0}^{2} d_{1}^{2}}{4}\left\langle I_{3}\right\rangle^{2}, \Delta \beta_{n}^{2}=\frac{1}{d_{1}^{2}}+\Delta \beta_{3}^{2}$. So, the angular spectrum of the product $I_{e}$ is composed of two parts:

$$
S_{I_{e}}=S_{p}(\beta)+S_{b}(\beta)
$$

where

$$
S_{p}(\beta)=2 \pi\left\langle I_{e}\right\rangle^{2} \int_{0}^{\infty} r \exp \left(-\frac{r^{2}}{2 d_{1}^{2}}\right) J_{0}(\beta r) \mathrm{d} r,
$$

and

$$
S_{b}(\beta)=2 \pi\left\langle I_{e}\right\rangle^{2} \int_{0}^{\infty} r \exp \left(-\frac{r^{2} \Delta \beta_{n}^{2}}{2}\right) J_{0}^{2}\left(\beta_{0} r\right) J_{0}(\beta r) \mathrm{d} r .
$$

The first part $S_{p}$ is an axial coherent peak and the second term $S_{b}$ is the background which gives rise to noise in the amplified signal. Besides the axial part, the spectral amplitude $S_{b}$ has a ring part at $\beta=2 \beta_{0}$, see Fig. 1. As we can see, the axial part of $S_{p}$ is much larger than the axial part of $S_{b}$. We note that at $z=0$ the integral powers of $S_{p}$ and $S_{b}$ are the same. Here and further the calculations were performed for the type II KTP crystal, and the refractive indices were taken from [11]: 


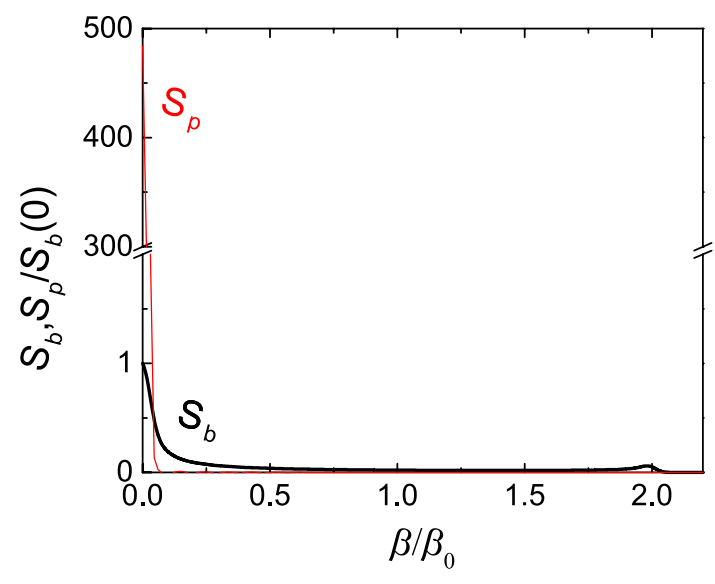

Fig. 1. Integrals $S_{p}$ and $S_{b}$, Eqs. (10) and (11). KTP type II crystal, $\theta_{0}=0.5^{\circ}, d_{1}=500 \mu \mathrm{m}, \Delta \beta_{3}=\beta_{0} / 50$.

$n_{1}=1.830$ (signal, o-wave), $n_{2}=1.747$ (idler, e-wave), and $n_{3}=1.788$ (pump, e-wave). The degenerate interaction at the pump wavelength $\lambda_{3}=0.532 \mu \mathrm{m}$ was assumed. The interaction takes place in the $x y$ plane and is nearly noncritical.

\subsection{Governing equations}

The nonlinear parametric interaction of the conical pump (index 3), axial signal (1) and conical idler (2) beams is described by the following equations for the fields:

$$
\begin{aligned}
& \frac{\partial A_{1}}{\partial z}+\frac{\mathrm{i}}{2 k_{1}} \Delta_{\perp} A_{1}=\sigma_{1} A_{2}^{*} A_{3} \exp (\mathrm{i} \Delta z), \\
& \frac{\partial A_{2}}{\partial z}+\frac{\mathrm{i}}{2 k_{2}} \Delta_{\perp} A_{2}=\sigma_{2} A_{1}^{*} A_{3} \exp (\mathrm{i} \Delta z), \\
& \frac{\partial A_{3}}{\partial z}+\frac{\mathrm{i}}{2 k_{3}} \Delta_{\perp} A_{3}=-\sigma_{3} A_{1} A_{2} \exp (-\mathrm{i} \Delta z) .
\end{aligned}
$$

Here $\Delta=\frac{\beta_{0}^{2}}{2}\left(\frac{1}{k_{2}}-\frac{1}{k_{3}}\right)$ is the phase mismatch, $\sigma$ is the nonlinear coupling coefficient, $\sigma \propto \omega_{j}, \omega_{j}$ is the cyclic frequency of the $j$ th wave, $k$ is the wavenumber, and $\Delta_{\perp}=\frac{\partial^{2}}{\partial x^{2}}+\frac{\partial^{2}}{\partial y^{2}}$. As mentioned earlier, the nearly noncritical interaction in the type II KTP crystal is studied. Therefore the walk-off terms are not included in the equations.

Further a theoretical consideration is performed under the assumption that the pump beam is not depleted. Moreover, we assume that the diffraction of the pump ring could be neglected. Then, the following condition should be fulfilled:

$$
\begin{aligned}
& \frac{\beta^{2}-\beta_{0}^{2}}{2 k_{3}} z \leq \frac{\Delta \beta_{3} \beta_{0} z}{k_{3}} \\
& =\frac{2 \Delta \beta_{3}}{\beta_{0}} \frac{z}{l_{p}} \ll 1, \text { where } l_{p}=\frac{2 k_{3}}{\beta_{0}^{2}} .
\end{aligned}
$$
by

In this case the field of the pump beam is described

$$
A_{3} \approx A_{30} \exp \left(\frac{\mathrm{i} \beta_{0}^{2} z}{2 k_{3}}\right) .
$$

The boundary condition for the signal beam is given by Eq. (5). The field of the input idler beam is given by

$$
A_{20}(x, y)=0
$$

and the input pump beam was simulated according to Ref. [8]:

$$
\begin{aligned}
& A_{30}(x, y)=\exp \left(-\left(x^{2}+y^{2}\right) / d_{3}^{2}\right) \\
& \times \sum_{n=1}^{N} U_{n}(x, y) \exp \left(\mathrm{i} \beta_{0} \cos \left(\varphi_{n}\right) x+\mathrm{i} \beta_{0} \sin \left(\varphi_{n}\right) y\right),
\end{aligned}
$$

where $\varphi_{n}=2 \pi(n-1) / N$ is the propagation angle of the $n$th intersecting beam and $d_{3}$ is the beam radius. Amplitudes $U_{n}$ were simulated according to Ref. [12] where the simulation of the Gaussian-Gaussian noise is described:

$$
U_{n}(x, y)=b_{0} \frac{1}{\sqrt{N_{s}}} \sum_{s=1}^{N_{s}} \exp \left(\mathrm{i} K_{x s} x+\mathrm{i} K_{y s} y+i \xi_{s}\right) .
$$

Here $K_{x s}$ and $K_{y s}$ are the random numbers with normal distribution and variance $\Delta \beta_{3} / \sqrt{2} . \xi_{s}$ is the uniformly distributed phase. $N_{s}$ has to be sufficiently large. Amplitude $b_{0}=a_{30} / \sqrt{N}$ and $a_{30}^{2}=\left\langle\left|A_{30}(0,0)\right|^{2}\right\rangle$.

We note that the pump field (16) is not homogeneous due to the Gaussian aperture. It obeys a conical angular spectrum [8]. In Fig. 2, we depict the numerically simulated pump beam profile (left) and the angular spectrum (center). Here, we used a rather large value of the ratio $\Delta \beta_{3} / \beta_{0}$ to make the ring in the angular spectrum visible. From the right figure one can see that the averaged profile (curve 2 ) coincides with the theoretical function (curve 3 ) given by the Gaussian exponent in Eq. (16):

$$
\left\langle\left|A_{30}\right|^{2}\right\rangle=a_{30}^{2} \exp \left(-2 \frac{\rho^{2}}{d_{3}^{2}}\right)
$$

Further we distinguish two cases: (i) the onset of amplification and (ii) exponential amplification. 

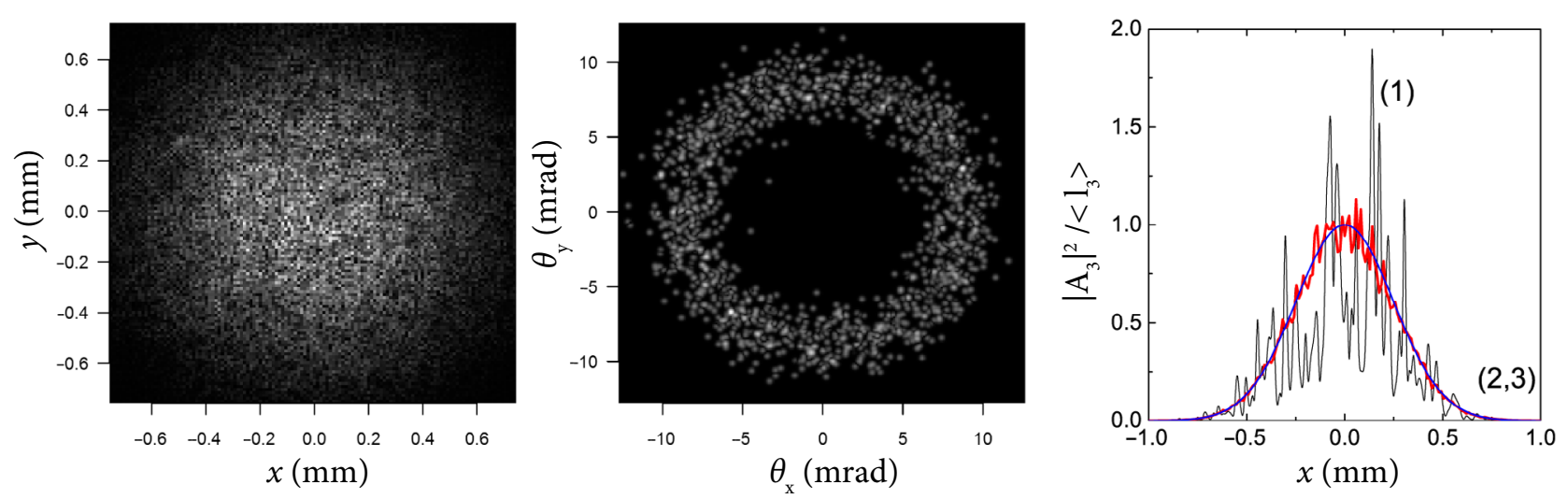

Fig. 2. Numerical simulation. Typical pump beam intensity distribution (left), profile (right) and angular spectrum (center). Right: intensity profile at $y=0$. One realization ( 1 , black), average of 200 realizations ( 2 , red online), theoretical function (3, blue online). Parameters: $d_{3}=500 \mu \mathrm{m}, \theta_{0}=0.5^{\circ}$ (inside the crystal), $\Delta \beta_{3} / \beta_{0}=0.2, N=32$.

\subsection{Onset of amplification}

In the case of a weak amplification the signal beam does not change considerably, so in the second equation of Eqs. (12) we can assume that $A_{1} \approx A_{10}$, where $A_{10}$ is given by Eq. (5). Then, for the idler beam we obtain

$$
\frac{\partial A_{2}}{\partial z}+\frac{\mathrm{i}}{2 k_{2}} \Delta_{\perp} A_{2}=\sigma_{2} A_{10}^{*} A_{30} \exp \left(\mathrm{i} \frac{\beta_{0}^{2}}{2 k_{2}} z\right)
$$

The idler beam is conical. Further we assume that the Rayleigh length of the input signal beam exceeds the length of a nonlinear crystal. In this case the diffraction of an idler ring can also be neglected. Then, we can write $A_{2}=V_{2} \exp \left(\mathrm{i} \frac{\beta_{0}^{2}}{2 k_{2}} z\right)$ and

$$
\begin{aligned}
& \frac{\partial V_{2}}{\partial z} \approx \sigma_{2} A_{10}^{*} A_{30}, \\
& A_{2} \approx \sigma_{2} z A_{10}^{*} A_{30} \exp \left(\mathrm{i} \frac{\beta_{0}^{2}}{2 k_{2}} z\right) .
\end{aligned}
$$

Then, for the signal beam we obtain

$$
\frac{\partial A_{1}}{\partial z}+\frac{\mathrm{i}}{2 k_{1}} \Delta_{\perp} A_{1}=\sigma_{1} \sigma_{2} z A_{10} I_{3}
$$

where $I_{3}=\left|A_{30}\right|^{2}$. So, the onset of amplification is mainly caused by pump beam intensity.

We have already studied the correlation properties of the right-hand expression in Subsect. 2.1. Its angular spectrum obeys both coherent and noise parts $\left(S_{p}\right.$ and $\left.S_{b}\right)$. Here, we will show that the noise part is filtered out during the propagation in a nonlinear crystal. Further, we perform the Hankel transform of Eq. (21) and obtain

$$
\frac{\partial S_{1}}{\partial z}-\frac{\mathrm{i} 2 p^{2}}{l_{p 1}} S_{1}=\sigma_{1} \sigma_{2} z f(p),
$$

where $S_{1}$ is the spectral amplitude of the signal beam, $l_{p 1}=4 k_{1} / \beta_{0}{ }^{2} \approx l_{p}, p=\beta / \beta_{0}$ and

$$
f(p)=2 \pi a_{0} \int_{0}^{\infty} r \exp \left(-\frac{r^{2}}{d_{1}^{2}}\right) I_{3} J_{0}\left(p \beta_{0} r\right) \mathrm{d} r .
$$

The solution of Eq. (22) reads

$$
\begin{aligned}
& S_{1}=\exp \left(\mathrm{i} \frac{2 p^{2} z}{l_{p}}\right) \\
& \times\left[S_{10}+\sigma_{1} \sigma_{2} f(p) \int_{0}^{z} z^{\prime} \exp \left(-2 \mathrm{i} p^{2} z^{\prime} / l_{p}\right) \mathrm{d} z^{\prime}\right] .
\end{aligned}
$$

Here, $S_{10}=S_{1}(z=0)$. Further we involve into consideration the nonlinear interaction length $L_{n}: L^{2}{ }_{n}=1 /$ $\sigma_{1} \sigma_{2}\left\langle I_{3}\right\rangle$. We assume that $z / L_{n}$ is small at the onset of the amplification and ignore small terms $z^{4} / L_{n}^{4}$ in the expression of $\left|S_{1}\right|^{2}$. After averaging, $\langle f(p)\rangle=\left\langle I_{3}\right\rangle$ $S_{10}(p)$, and for the parametric gain $\eta$ we obtain

$$
\eta=\frac{\left\langle\left|S_{1}\right|^{2}\right\rangle-S_{10}^{2}}{S_{10}^{2}}=\frac{2}{L_{n}^{2}} \int_{0}^{z} z^{\prime} \cos \left(2 p^{2} z^{\prime} / l_{p}\right) \mathrm{d} z^{\prime}
$$

At $p=0$ we find $\eta=\frac{z^{2}}{L_{n}^{2}}$. From the condition $\eta=\frac{1}{2} \frac{z^{2}}{L_{n}^{2}}$ we find the FWHM bandwidth of the OPA (at $z \ll L_{n}$ ):

$$
\frac{\Delta \beta_{\mathrm{OPA}}}{\beta_{0}}=\sqrt{\frac{\pi}{4}} \sqrt{\frac{l_{p}}{z}} .
$$

Therefore, the bandwidth decreases with a propagation in the nonlinear crystal. If $l_{p}<z \ll L_{n}$, one can 
observe the filtering of noise in the signal beam. That is caused by a specific angular spectrum of pump beam intensity $\left(S_{b}\right.$ in Fig. 1). At $z \gg l_{p}$ the influence of pump beam incoherence on the angular spectrum of the signal beam should be negligible. In this case, a coherent parametric amplification in the field of an incoherent pump beam becomes possible. We note that at $\beta_{0} \gg \Delta \beta_{3}$ the parameter $l_{p}$ denotes an influence of conical pump beam incoherence in parametric amplification.

Further, we simulated numerically nonlinear coupling equations (12) by the use of the Fourier split-step method [13]. In order to investigate the onset of signal amplification we have chosen the parameters given in the caption of Fig. 3. In this case the condition of Eq. (13) is satisfied: $2 \Delta \beta_{2} z / \beta_{0} l_{p}=0.23$ (at $z=18 \mathrm{~mm}$ ) and according to Eq. (26) $\Delta \beta_{\mathrm{OPA}} / \beta_{0}=0.12$. Therefore, the diffraction of the pump beam can be neglected and the filtering of noise should be observed, see Fig. 1. Indeed, as we can see from Fig. 3, the noisy structure is added to the signal beam due to amplification (center graph). The correlation radius increases when the propagation distance $z$ is much larger than $l_{p}=318 \mu \mathrm{m}$ (right graph). Note that here $z<L_{n}$ and the amplification is weak. Therefore, we depict the difference of the intensities of amplified and input beams (center and right graphs).

\subsection{Exponential amplification}

We intend to amplify the signal beam considerably. Let us assume that at $z \gg L_{n}$ the exponential amplification of the signal beam starts. Therefore, we write for the signal angular spectrum at $\Gamma z \gg 1$

$$
\left\langle S_{1}\right\rangle=\exp (\Gamma z)\left\langle S_{10}(\beta)\right\rangle,
$$

where the increment $\Gamma$ has to be defined. We express the spectral amplitude of the idler beam as

$$
S_{2}=\exp \left(\mathrm{i} \beta_{0}{ }^{2} z / 2 k_{2}\right) B_{2}(\beta, z) \text {. }
$$

Let us assume a narrow ring for the idler beam, so that $S_{2} \neq 0$ at $\beta \approx \beta_{0}$. Then, we obtain the equation

$$
\frac{\partial B_{2}}{\partial z}=\sigma_{2} F T 2\left(A_{1}^{*}(x, y, z) A_{30}(x, y)\right),
$$

and the solution

$$
\begin{aligned}
& S_{2}=\sigma_{2} \exp \left(\mathrm{i} \beta_{0}^{2} z / 2 k_{2}\right) \\
& \times \int_{0}^{z} \mathrm{~d} z^{\prime} F T 2\left(A_{1}^{*}\left(x, y, z^{\prime}\right) A_{30}(x, y)\right) .
\end{aligned}
$$

FT2(•) denotes the two-dimensional Fourier transform. The field of the idler beam is given by

$$
\begin{aligned}
& A_{2}=\sigma_{2} \exp \left(\mathrm{i} \beta_{0}^{2} z / 2 k_{2}\right) \\
& \times A_{30}(x, y) \int_{0}^{z} \mathrm{~d} z^{\prime} A_{1}^{*}\left(x, y, z^{\prime}\right) .
\end{aligned}
$$

We insert this result into the first equation of Eqs. (12) and make use of Eqs. (14) and (27). We obtain

$$
\Gamma\left\langle S_{10}\right\rangle-\frac{\mathrm{i} \beta^{2}}{2 k_{1}}\left\langle S_{10}\right\rangle=\frac{1}{L_{n}^{2} \Gamma} S_{13}
$$

where $S_{13}=F T 2\left(\left\langle A_{10}(x, y)\right\rangle\left\langle f_{3}(x, y)\right), f_{3}=\left|A_{30}\right|^{2} / a_{30}^{2}\right.$. Here, we assumed that $\left.F T 2\left(\left\langle A_{1}\right\rangle f_{3}\right\rangle\right)=\exp (\Gamma z) F T 2\left(\left\langle A_{10}\right\rangle\right.$ $\left.\left\langle f_{3}\right\rangle\right)$, where $A_{10}$ is the inverse two-dimensional Fourier transform of $S_{10}$. We also assumed that signal and pump beams are uncorrelated: $\left\langle A_{1_{3}}\right\rangle=\left\langle A_{1}\right\rangle\left\langle f_{3}\right\rangle$. The second assumption is correct in the case of a small nonlinearity with respect to the diffraction term [14, 15,. Note that
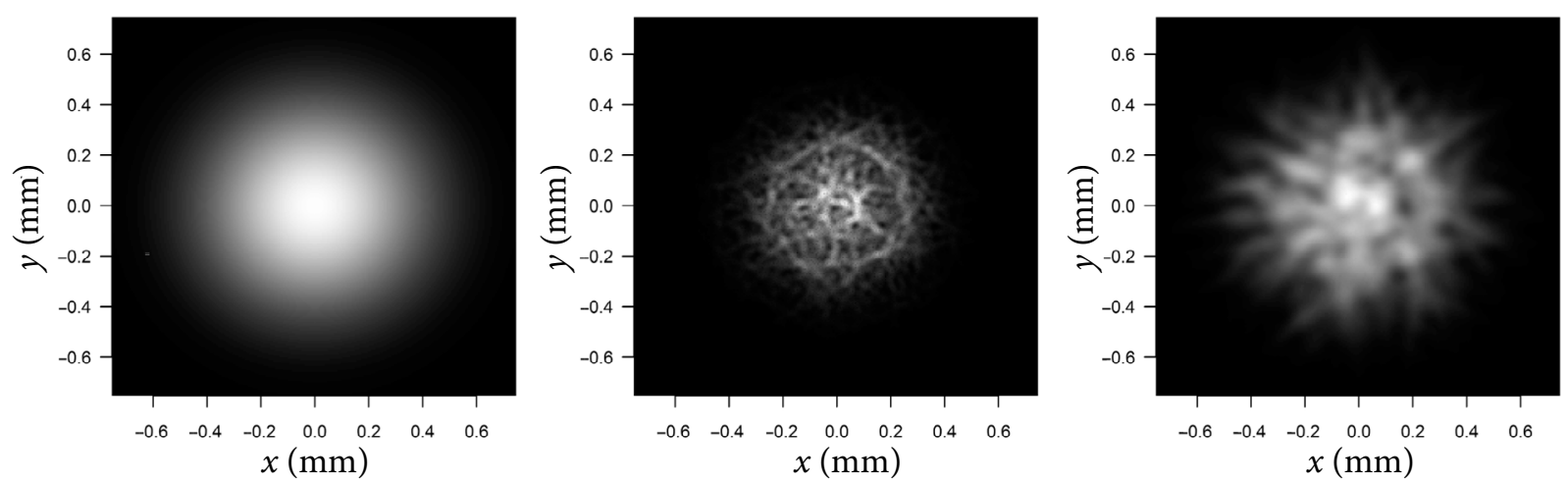

Fig. 3. Numerical simulation of Eqs. (12). Signal beam intensity distributions at the onset of amplification. Left: intensity distribution of the input signal beam $\left|A_{10}\right|^{2}$. Difference $\left|A_{1}\right|^{2}-\left|A_{10}\right|^{2}$ at $z=1.8 \mathrm{~mm}$ (center) and $z=18 \mathrm{~mm}$ (right). One realization. Parameters: $d_{1}=d_{3}=500 \mu \mathrm{m}, \theta_{0}=1^{\circ}$ (inside the crystal), $\Delta \beta_{3} / \beta_{0}=0.002, N=256, L_{n}=20 \mathrm{~mm}$, $a_{0} / a_{30}=10^{-4}$. 
$\left\langle f_{3}\right\rangle$ is given by the Gaussian exponent $\left\langle f_{3}\right\rangle=\exp \left(-2 \rho^{2} /\right.$ $d_{3}^{2}$ ) (Eq. (18) and Fig. 2, right).

First, we simulated Eqs. (12) for the same input pump beam as in Fig. 2. In order to examine the exponential amplification we set $z / L_{n}=4$. The output signal and idler angular spectra are depicted in Fig. 4 (top). As we can see, the idler beam obeys a conical angular spectrum. It is incoherent. The signal angular spectrum consists of a narrow central spot. Although the angular spectrum of the signal beam is narrow, the incoherent structure becomes visible in the intensity distribution (Fig. 4, bottom).
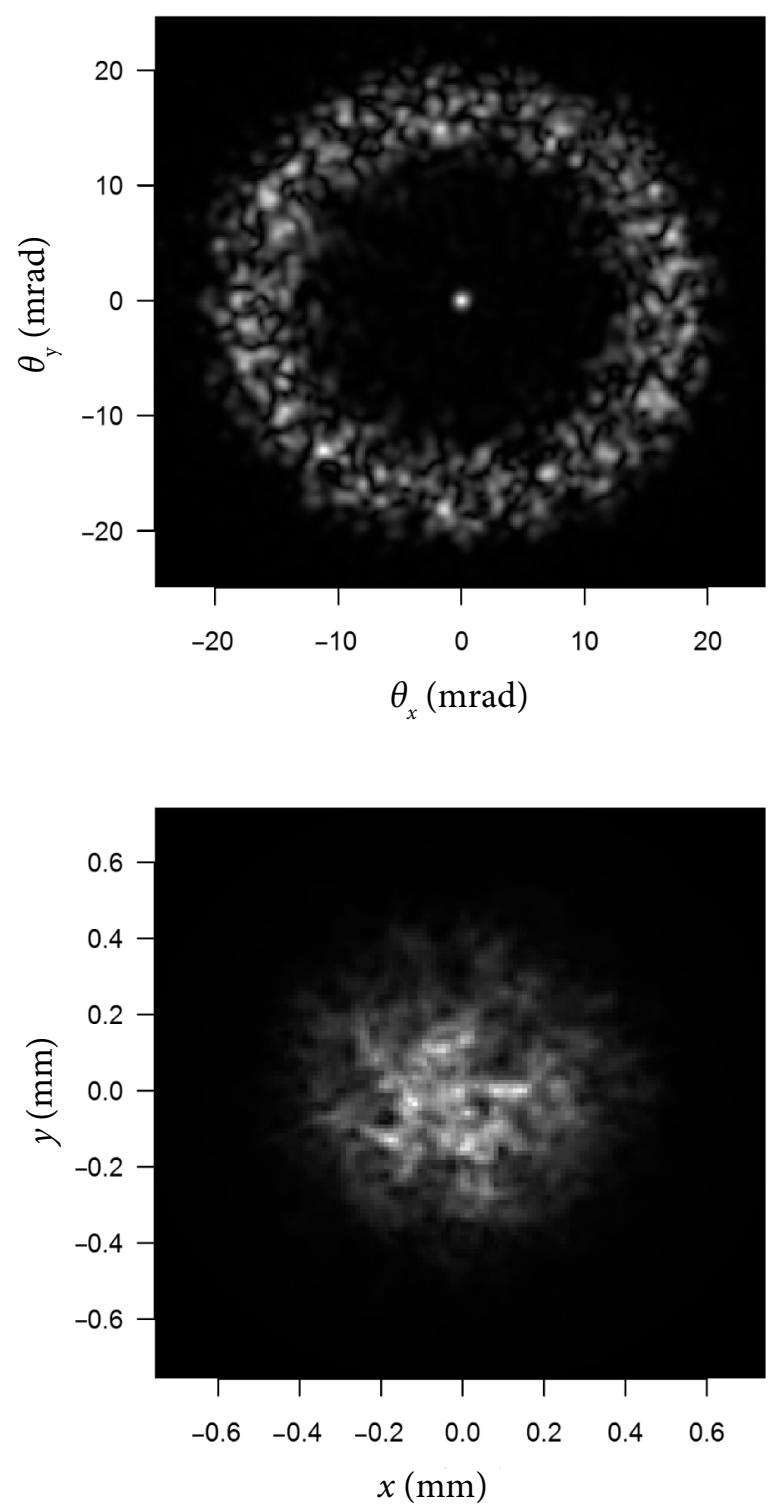

Fig. 4. Numerical simulation of Eqs. (12). Top: output signal (axial) and idler (conical) angular spectra. Bottom: output signal intensity distribution. Input parameters are the same as in Fig. $1 . d_{1}=d_{3}, L_{n}=2.5 \mathrm{~mm}$, $z=10 \mathrm{~mm}, a_{0} / a_{30}=10^{-4}$. One simulation.
As we can see from Eq. (32), at $\beta=0$, $\Gamma= \pm \sqrt{\left\langle S_{13}(0)\right\rangle /\left\langle S_{10}(0)\right\rangle} / L_{n}$. Therefore, the increment of the central part of the spectrum coincides with the increment which is obtained in the case of the plane wave pump $\left(\Gamma=1 / L_{n}\right)$ if $d_{3} \gg d_{1}$, and we can approximately write $\left\langle S_{13}\right\rangle \approx\left\langle S_{10}\right\rangle$. Then, the amplification of the narrowband signal beam in the field of the incoherent conical beam is the same as in the field of the plane wave. In order to check this result, we performed numerical simulations of Eqs. (12) in the case when the ratio of the radius of signal and pump beams is $d_{1} / d_{3}=0.25$. The numerically calculated growth of the signal spectral amplitude was compared with the following formula:

$$
\left\langle S_{1}(0)\right\rangle=\left\langle S_{10}(0)\right\rangle \cosh \left(\frac{z}{L_{n}}\right) .
$$

Here $S_{10}$ is the input spectral amplitude. We used the function $\cosh (\bullet)$ instead of $\exp (\bullet)$ since the increment $\Gamma$ obeys both positive and negative values and $\partial S_{1} / \partial z=0$ at $\beta=0, z=0$. The results are presented in Fig. 5. We see that a good coincidence of the theoreti$\mathrm{cal}$ and numerical data is obtained (curves 1 and 2). Furthermore, as we can see from Fig. 6(a), the initial angular profile is preserved in this case. Curve 3 in Fig. 5 and curves 1, 2 in Fig. 6 (b) correspond to another case when the beam radii of the input pump and signal coincide. Then, the increment is smaller, and the angular profile of the signal beam becomes broader due to the amplification in the field of a nonhomogeneous pump beam envelope.

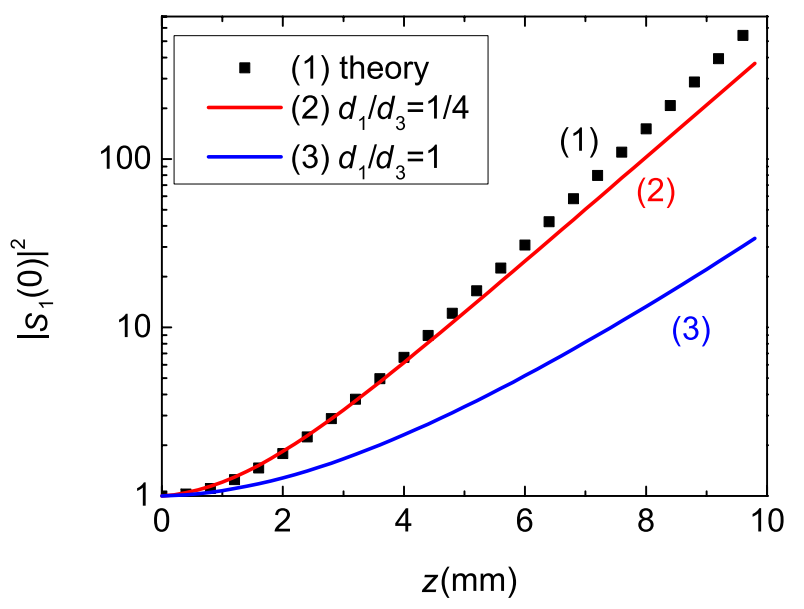

Fig. 5. Numerical simulation of Eqs. (12). Amplification of the spectrum radiance of the signal beam: theoretical (1) and numerical $(2,3)$ curves. $d_{1} / d_{3}: 0.25(2), 1$ (3). Parameters: $d_{3}=500 \mu \mathrm{m}, \theta_{0}=0.5^{\circ}$ (inside the crystal), $\Delta \beta_{3} / \beta_{0}=0.2, N=32, L_{n}=2.5 \mathrm{~mm}, a_{0} / a_{30}=10^{-4}$. Average of 50 simulations. 

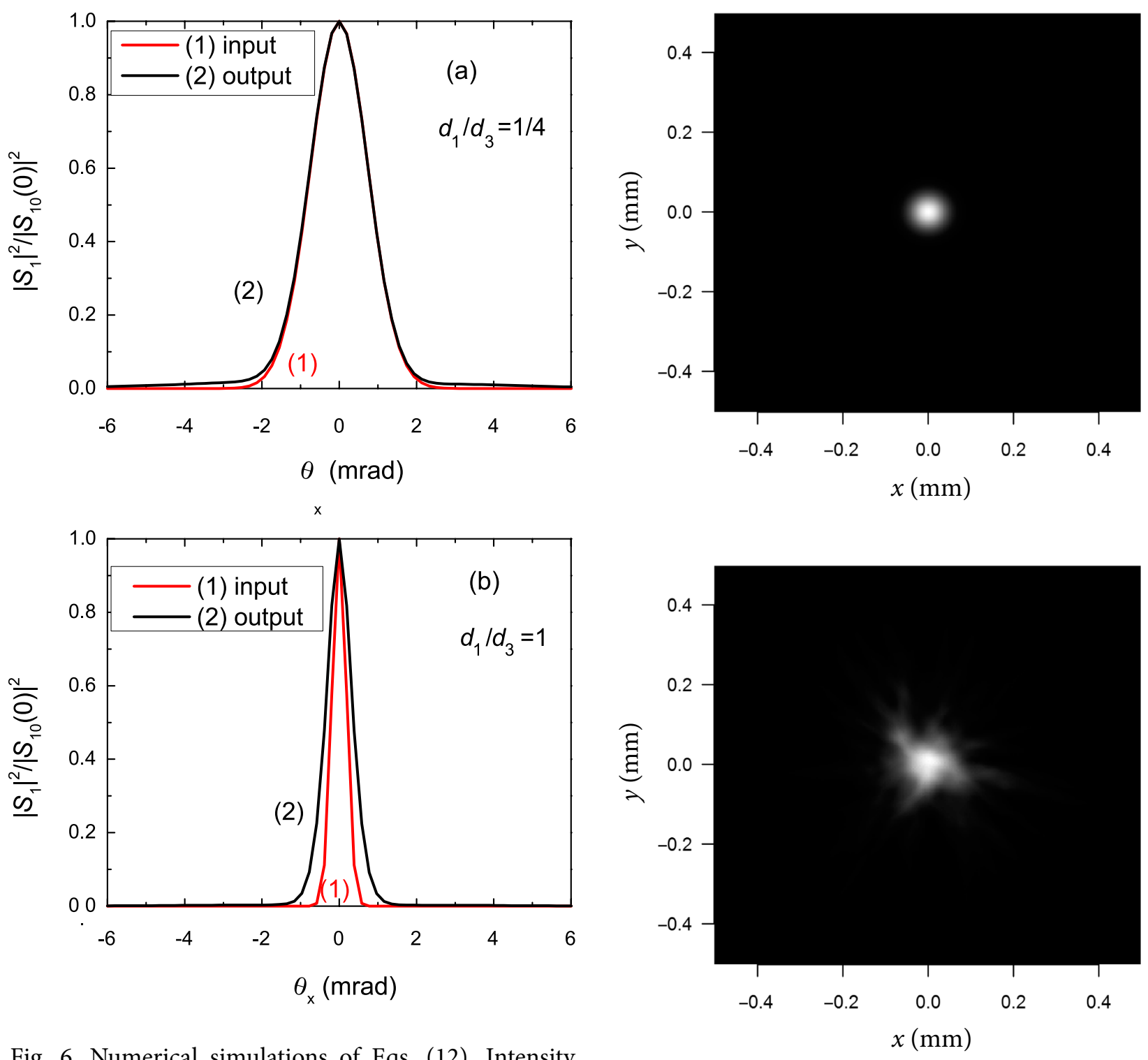

Fig. 6. Numerical simulations of Eqs. (12). Intensity profiles of the input seed (1) and amplified signal (2) at $z=10 \mathrm{~mm} . d_{1} / d_{3}: 0.25(\mathrm{a}), 1(\mathrm{~b})$. Parameters the same as in Fig. 4. Average of 50 simulations.

If we intend to amplify a narrowband signal beam without distortions in the intensity profile (see Fig. 4, bottom), the parameters should be set that would provide the noise filtering (see Fig. 3). The parameters given in the caption of Fig. 7 provide the combination of the filtering with the exponential amplification. In this case, the output signal intensity profile obeys less distortions and the amplification of the spectral radiance is similar to the one depicted in Fig. 5, curve 2. The parameters yield a smaller value of $l_{p}$ (because of larger $\theta_{0}$ ), so that $l_{p}=L_{n}$. In order to avoid pump beam diffraction, we set smaller $\Delta \beta_{3}$. Therefore, the coherent parametric amplification of the narrowband signal beam in the field of the conical incoherent beam is possible.

Fig. 7. Numerical simulation of Eqs. (12). Intensity profiles of input (top) and amplified signal (bottom). Parameters: $d_{3}=500 \mu \mathrm{m}, d_{1} / d_{3}=0.1, \theta_{0}=1.5^{\circ}$ (inside the crystal), $\Delta \beta^{3} / \beta_{0}=0.02, N=256, L_{n}=2.5 \mathrm{~mm}, z=10 \mathrm{~mm}$, $a_{0} / a_{30}=10^{-4}$. One simulation.

\section{Experimental results}

The experiment on parametric amplification pumped by incoherent radiation was performed using a diode-pumped Nd:YAG laser system (APL 2210B, EKSPLA). The laser produced 50 ps pulses at the $1 \mathrm{kHz}$ repetition rate and a near-Gaussian spatial beam profile. The experimental set-up is presented in Fig. 8 (left). The OPA was pumped by the second harmonic of the Nd:YAG laser (wavelength $\lambda_{3}=532 \mathrm{~nm}$ ) and seeded by the fundamental harmonic of the same laser. In order to obtain 

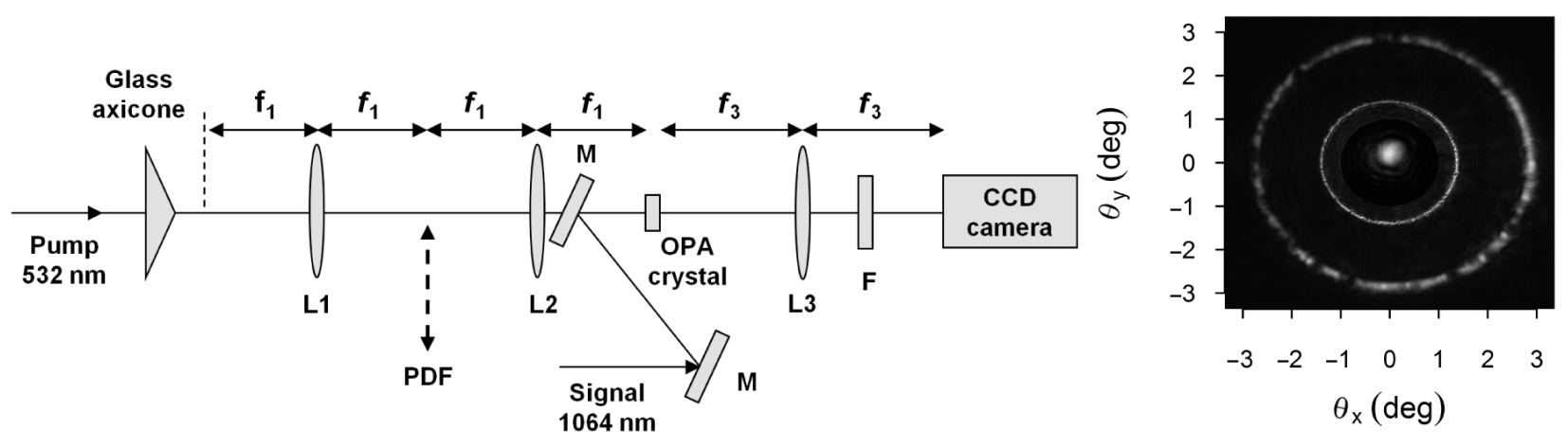

Fig. 8. Experimental set-up (left): L1-L3, lenses; M, dielectric mirrors; F, filter; PDF, phase-distorting film. Output spectra (right) of signal (central spot), idler (outer ring) and pump (inner ring) beams.

an incoherent conical pump radiation, a Bessel beam was formed initially using a high quality glass axicon with the cone angle equal to $0.067 \mathrm{rad}\left(\theta_{0} \approx 1^{\circ}\right.$ inside the crystal). The beam radius before the axicon was $800 \mu \mathrm{m}$ and the parameter defining quality of the Bessel beam (ratio of ring radius to ring width) was $\sim 170$. The Bessel beam formed behind the axicon was imaged onto the OPA crystal by two plano-convex lenses L1 and L2 with equal focal lengths of $f_{1}=100 \mathrm{~mm}$. Spatial incoherence of the conical beam was obtained by placing a phase-distorting film PDF (made of polyethylene) in the focal plane of lens L1 and the angular spectrum of the incoherent conical beam is presented in Fig. 8 (right, inner ring). The energy of the pump pulse up to $800 \mu \mathrm{J}$ was adequate to amplify the seed in a $10 \mathrm{~mm}$ long KTP crystal. The signal beam was injected into OPA and type II interaction close to the noncritical phase-matching (KTP crystal orientation $\theta=0, \varphi=23.2^{\circ}$ ) was realized. In this experiment, the generated incoherent conical pump beam can be viewed as a set of intersecting beams with uncorrelated phases due to the fact that the PDF was placed at the far field (ring) of the conical beam. So, the incoherent conical beam was formed.

First, spectral features were investigated. A far field of the OPA output was imaged by lens $\mathrm{L} 3\left(f_{3}=150 \mathrm{~mm}\right)$ onto the CCD camera and the angular spectra of propagating beams were registered. Filter F was used to suppress the pump power. Angular spectra of output beams are presented in Fig. 8 (right) where the central spot and the outer ring correspond to the amplified signal and generated idler beams, respectively. An intensity modulated inner ring is the angular spectrum of the spatially incoherent conical pump beam. Note that an outer ring is also modulated. The asymmetry of rings with respect to the central spot is due to the spatial walk-off of extraordinary polarized waves.
In Fig. 9 (a) the dependence of parametric gain on the input pump energy is presented. Signal energy increases exponentially and further scaling of pump energy was prevented by optical damage to the crystal. Intensity profiles of input and amplified signal beams
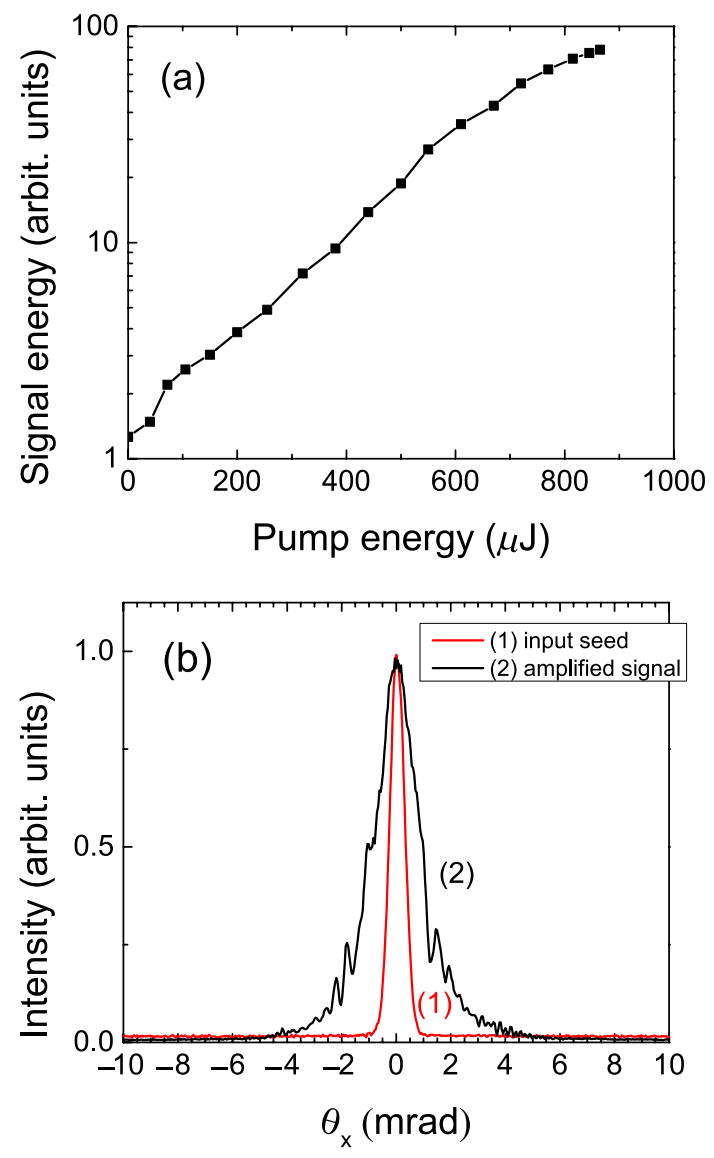

Fig. 9. Experimental data. (a) Dependence of parametric gain of signal beam on pump energy. (b) Angular spectrum of input (1) and amplified (2) signal beam. Pump energy: $270 \mu \mathrm{J}(2)$. 
are presented in Fig. 9(b), and curves 1, 2 reveal that the input narrowband angular spectrum becomes broader during the amplification. This behaviour confirms the theoretical results presented in Fig. $6(\mathrm{~b})$.

\section{Conclusions}

The optical parametric amplification of the signal beam by the spatially incoherent conical pump beam is demonstrated. The acquired noise of the signal beam in the onset of amplification in the field of the incoherent conical beam is filtered during the propagation in the nonlinear medium. At $z \gg l_{p}$ the influence of pump beam incoherence on the angular spectrum of the signal becomes negligible but can be observed in the intensity profile of the signal beam. The distortions of signal beam intensity gradually disappear when the pump cone angle $\theta_{0}$ increases (pump incoherence parameter $l_{p} \propto \theta_{0}^{-2}$ decreases).

When the radius of the signal beam is considerably smaller than the radius of the pump beam envelope $\left(d_{1} \ll d_{3}\right)$, an exponential parametric amplification takes place. In this case, the initial angular profile of the signal beam is preserved during amplification. If $d_{1} \geq d_{3}$, the angular profile of the signal beam becomes broader due to amplification in the field of the nonhomogeneous envelope of the pump beam.

A good qualitative agreement between theoretical and experimental data was obtained. The possibility to amplify significantly a narrowband signal without considerable distortions in the intensity profile is demonstrated by the use of numerical simulations.

\section{References}

[1] A. Piskarskas, V. Pyragaite, and A. Stabinis, Generation of coherent waves by frequency up-conversion and down-conversion of incoherent light, Phys. Rev. A 82(5), 053817 (2010), http://dx.doi. org/10.1103/PhysRevA.82.053817

[2] A. Picozzi and M. Haelterman, Parametric threewave soliton generated from incoherent light, Phys. Rev. Lett. 86(10), 2010-2013 (2001), http:// dx.doi.org/10.1103/PhysRevLett.86.2010

[3] V. Pyragaite, A.Stabinis, A. Piskarskas, and V. Smilgevičius, Parametric amplification in the field of incoherent light, Phys. Rev. A 87(6), 063809 (2013), http://dx.doi.org/10.1103/PhysRevA.87.063809
[4] V. Pyragaite, V. Smilgevičius, R. Butkus, A. Stabinis, and A. Piskarskas, Conversion of broadband incoherent pump to narrowband signal in an optical parametric amplifier, Phys. Rev. A 88(2), 023820 (2013), http://dx.doi.org/10.1103/ PhysRevA.88.023820

[5] C. Sun, S. Jia, C. Barsi, S. Rica, A. Picozzi, and J.W. Fleischer, Observation of the kinetic condensation of classical waves, Nat. Phys. 8, 470-474 (2012), http://dx.doi.org/10.1038/nphys2278

[6] J. Durnin, Exact solutions for nondiffracting beams. I. The scalar theory, I. Opt. Soc. Am. A 4(4), 651-654 (1987), http://dx.doi.org/10.1364/ JOSAA.4.000651

[7] D. McGloin and K. Dholakia, Bessel beams: Diffraction in a new light, Contemp. Phys. 46(1), 15-28 (2005), http://dx.doi. org/10.1080/0010751042000275259

[8] P. Stanislovaitis, A. Narmontas, V. Pyragaite, and V. Smilgevičius, Generation of a coherent secondharmonic beam from incoherent conical beams, Phys. Rev. A 89(4), 043821 (2014), http://dx.doi. org/10.1103/PhysRevA.89.043821

[9] R. Gadonas, A. Marcinkevičius, A. Piskarskas, V. Smilgevičius, and A. Stabinis, Travelling wave optical parametric generator pumped by a conical beam, Opt. Commun. 146(1-6), 253-256 (1998), http://dx.doi.org/10.1016/S0030-4018(97)00559-2

[10] V. Pyragaite, V. Smilgevičius, R. Butkus, A. Narmontas, A. Stabinis, and A. Piskarskas, Controlling the signal angular profile in a Bessel-beampumped optical parametric amplifier, Phys. Rev. A 90(2), 023807 (2014), http://dx.doi.org/10.1103/ PhysRevA.90.023807

[11] A.V. Smith, Computer Code SNLO (AS-Photonics, Albuquerque, NM, 2012).

[12]R. Loudon, The Quantum Theory of Light (Oxford University Press, New York, 2000).

[13] G.P. Agrawal, Nonlinear Fiber Optics (Academic Press, San Diego, CA, 1989).

[14] A. Picozzi and P. Aschieri, Influence of dispersion on the resonant interaction between three incoherent waves, Phys. Rev. E 72(4), 046606 (2005), http://dx.doi.org/10.1103/PhysRevE.72.046606

[15] A. Picozzi, J. Garnier, T. Hansson, P. Suret, S. Randoux, G. Millot, and D.N. Christodoulides, Optical wave turbulence: Towards a unified nonequilibrium thermodynamic formulation of statistical nonlinear optics, Phys. Rep. 542(1), 1-132 (2014), http://dx.doi.org/10.1016/j.physrep.2014.03.002 


\title{
ŠVIESOS PARAMETRINIS STIPRINIMAS KAUPINANT NEKOHERENTINIU KÜGINIU PLUOŠTU
}

\author{
V. Tamulienė, V. Smilgevičius, D. Kudarauskas, R. Butkus, A. Stabinis, A. Piskarskas
}

Vilniaus universiteto Kvantines elektronikos katedra, Vilnius, Lietuva

\section{Santrauka}

Pademonstruotas erdviškai koherentinio šviesos pluošto parametrinis stiprinimas kaupinant ji erdviškai nekoherentiniu kūginiu pluoštu. Nustatytos sąlygos, kada stiprinamo pluošto kampinis spektras lieka nepa-

kitęs. Tada stiprinimas vyksta pagal eksponentinị dèsnị ir kaupinimo pluoštas veikia kaip plokščia banga.

Gautas geras teorinių ir eksperimentinių rezultatų sutapimas. 